\title{
Optomechanics of a stable diffractive axicon light sail
}

\author{
Prateek R. Srivastava ${ }^{\mathrm{a}}{ }^{\mathbb{D}}$, Grover A. Swartzlander Jr. ${ }^{\mathrm{b}}{ }_{(\mathbb{D})}$ \\ Chester F. Carlson Center for Imaging Science, Rochester Institute of Technology, Rochester, NY 14623, \\ USA
}

Received: 24 February 2020 / Accepted: 17 June 2020 / Published online: 14 July 2020

(C) The Author(s) 2020

\begin{abstract}
Beamed propulsion of a light sail based on radiation pressure benefits from a passively self-stabilizing "beam riding" diffractive film. We describe the optomechanics of a rigid non-spinning light sail that mitigates catastrophic sail walk-off and tumbling by use of a flat axicon diffraction grating. A linear stability analysis and numerical integration of the coupled translational and rotational equations of motion are examined. Stability is traded against longitudinal acceleration. The examined system achieves $90 \%$ of the theoretical longitudinal force limit and stability against a relative sail translation up to $30 \%$ of the sail radius when the payload is attached to a long boom.
\end{abstract}

\section{Introduction}

Optical momentum carried by light [1] may be redirected by means of reflection, diffraction, scattering, or absorption to achieve radiation pressure on a material body. The idea of propelling a spacecraft to high velocities using solar radiations was first proposed in the early Twentieth century by Tsiolkovsky [2]. With the advent of lasers in the 1960s, laserpropelled sails reaching relativistic velocities for interstellar travel were proposed [3-13]. One of the many challenges associated with a laser-propelled light sail is the achievement of "beam-riding," i.e., the autonomous ability to remain in the beam path without tumbling or sliding out. A decades-long approach considers shaped mirror structures [14-24]. The ponderomotive (or gradient) force as used in optical tweezers [25] is currently negligible for practical space systems. The proposed use of diffraction to impart optical momentum to a body [26] provides an opportunity to decouple the sail shape from the momentum transfer process, thereby affording a new degree of design latitude. For example, a one-dimensional bi-grating has been explored to demonstrate the principle of self-stability [27,28]. The first experimental verification of this principle, along with the measurements of parametric damping, was reported by Chu [29,30]. Furthermore, advancements in the design and fabrication of diffractive films using metamaterial principles provide opportunities to engineer desired optomechanical and other properties into the functionality of a sail [31-44]. In the near term we envision the integration of diffractive light sail components on future solar sailing missions to help resolve engineering challenges [45] such as attitude control (see for example, Near-Earth Asteroid Scout [46], Solar Polar Imager [47], and Solar Cruiser [48]).

\footnotetext{
a e-mail: prs7786@g.rit.edu

b e-mail: grover.swartzlander@gmail.com (corresponding author)
} 
This report extends our one-dimensional theoretical investigations of a bi-grating to a two-dimensional axicon grating sail. Section 2 describes the transfer of momentum from the light beam to the sail, making use of sail and observer reference frames moving at small relative velocities, so that the Doppler-shifted wavelength may be ignored. The equations of motion for linear and angular degrees of freedom owing to optomechanical force and torque are described in Sect. 3. A linear stability analysis is described in Sect. 4 where conditions for stable light propulsion are described. Numerical solutions of the equations of motion are presented in Sect. 5, including an analysis of motion in the stable regime. Important findings are summarized in Sect. 6.

\section{Photon momentum transfer to a diffractive sail}

Let us consider a laser beam of characteristic radial width $w$ incident upon a sail of radius $a$. Radiation pressure applies a local force at all sail points, resulting in longitudinal acceleration along the optical axis, lateral force, and torque. The minimum beam size, $w_{0}$ (the waist), is positioned at the origin of the observer coordinate system $(X, Y, Z)$, as illustrated in Fig. 1, and the beam propagates in the $Z$-direction (the optical axis). The electric field profile of a monochromatic beam of wavelength $\lambda$ and constant power $P$ may be expressed [49]

$$
\begin{aligned}
E(X, Y, Z)= & \sqrt{I_{0}(Z)}\left(w_{0} / w(Z)\right) \exp \left(-\left(X^{2}+Y^{2}\right) / w(Z)^{2}\right) \exp (i \Phi(r, z)) \\
& \times \exp \left(i\left(k_{Z} Z-\omega t\right)\right)
\end{aligned}
$$

where $k_{Z}=2 \pi / \lambda, \omega=c k$ is the angular frequency, $c$ is the speed of light, $I_{0}(Z)=$ $2 P / \pi w^{2}(Z)$ is the irradiance on the optical axis, $w(Z)=w_{0}\left[1+\left(Z / Z_{0}\right)^{2}\right]^{1 / 2}$ is the radial beam size, $Z_{0}=\pi w_{0}^{2} / \lambda$ is the diffraction length, $\Phi(r, z)=k_{z}\left(X^{2}+Y^{2}\right) / 2 R(z)-$ $\arctan \left(Z / Z_{0}\right)$, and $R(Z)=Z\left[1+\left(Z_{0} / Z\right)^{2}\right]$ for a $\mathrm{TEM}_{00}$ Gaussian beam. Assuming the beam is much larger than the wavelength $\left(w_{0}>>\lambda\right)$, we ignore the transverse component of the wave vector, $k_{X}=\partial \Phi / \partial X$ and $k_{Y}=\partial \Phi / \partial Y$, which are much smaller than $k_{Z}$. That is, the paraxial approximation is made such the incident wave vector may be expressed $\vec{k}_{i}=(2 \pi / \lambda) \hat{Z}$.

We consider a sail comprised of a reflection grating that diffracts light toward the sail axis when illuminated at normal incidence. That is, the sail functions as an optical axicon (see inset of Fig. 1), having a periodic phase profile, $\Phi_{\text {axicon }}\left(\rho^{\prime}+\Lambda\right)=\Phi_{\text {axicon }}\left(\rho^{\prime}\right)=-2 \pi\left(\rho^{\prime} / \Lambda\right)$ where $\rho^{\prime}=\left(x^{\prime 2}+y^{\prime 2}\right)^{1 / 2}$. For analytical convenience we assume a single diffraction order, noting that this analysis may be readily extended to include multiple reflection and transmission orders. The axicon grating vector $\vec{K}$ lies in the plane of the sail and points radially toward the sail axis (see inset of Fig. 1).

The grating vector (see inset of Fig. 1) of the sail is directed radially inward from the center of the sail and is expressed

$$
\vec{K}=-(2 \pi / \Lambda)\left(\cos \psi \hat{x}^{\prime}+\sin \psi \hat{y}^{\prime}\right)
$$

where $\Lambda$ is the grating period and $\psi$ is the polar angle measured counterclockwise from $\hat{x}^{\prime}$. At normal incidence, the angle between $\hat{Z}$ and $\hat{z}$ is zero, i.e., the sail normal and incident wave vector are perfectly aligned and the grating functions as a reflective axicon.

For an arbitrary attitude, the momentum imparted to the sail may be determined from the difference of linear photon momenta before and after diffraction. This difference is quantified 


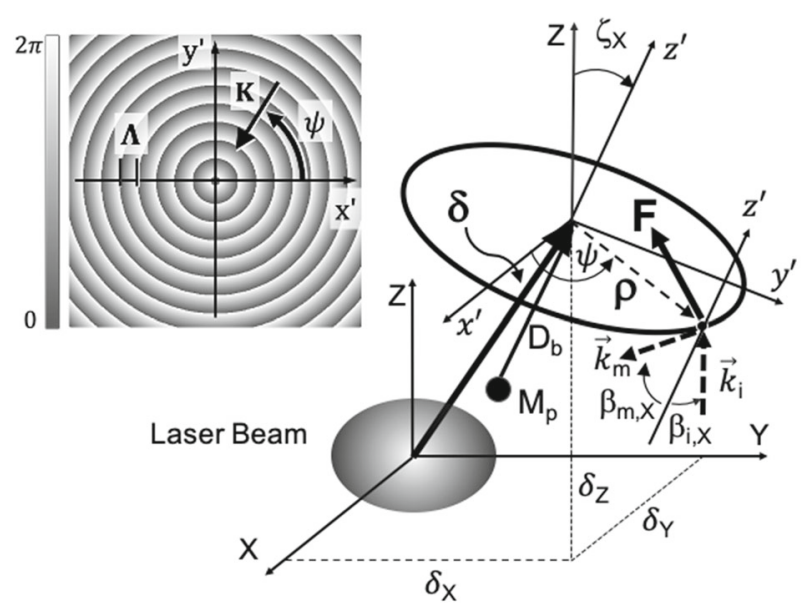

Fig. 1 Diffractive sail illuminated by a beam at incident angle $\beta_{i, X}$ and diffraction angle $\beta_{m, X}$. Sail tilt axis $\zeta_{X}=-\beta_{i, X}$. Attached payload of mass $M_{p}$ with boom length $D_{b}$. Laser beam origin $(X, Y, Z)=(0,0,0)$. Sail displacement $\vec{\delta}$. Inset: magnified view of axicon phase with grating vector $\vec{K}$

by the photon momentum transfer efficiencies in the two reference frames:

$$
\begin{aligned}
& \vec{\eta}^{\prime}=\left(\vec{k}_{i}^{\prime}-\vec{k}_{d}^{\prime}\right) /(2 \pi / \lambda) \\
& \vec{\eta}=\left(\vec{k}_{i}-\vec{k}_{d}\right) /(2 \pi / \lambda)
\end{aligned}
$$

where $\vec{k}_{i}^{\prime}\left(\vec{k}_{i}\right)$ is the incident wave vector in the sail frame (stationary frame) and $\vec{k}_{d}^{\prime}\left(\vec{k}_{d}\right)$ is the diffracted wave vector in the sail frame (stationary frame). For example, if $\vec{k}_{d}=-\vec{k}_{i}=$ $-(2 \pi / \lambda) \hat{Z}$ then $\vec{\eta}=2 \hat{Z}$. We note that for a Doppler-free elastic process $\left|\vec{\eta}^{\prime}\right|=|\vec{\eta}|$. For an arbitrary sail attitude the method of Euler angles is used to relate the wave vectors in the two reference frames (see "Appendix 1"). However, it is instructive to first consider a sail that is tipped in a single direction as depicted in Fig. 1.

Let us therefore set $\zeta_{Y}=\zeta_{Z}=0$ and consider a rotation angle $\zeta_{X}$ about the $\hat{X}$ axis. The angle $\zeta_{X}$ represents the attitude of the sail normal $\left(\hat{z^{\prime}}\right)$ with respect to the beam axis $(\hat{Z})$ and is measured counterclockwise from $\hat{Z}$, i.e., $\zeta_{X}<0$ for the attitude of sail shown in Fig. 1. The angle of incidence $\beta_{i, x}$ is measured counterclockwise from the sail normal such that $\beta_{i, x}=-\zeta_{X}$ and $\beta_{i, x}>0$ for the orientation shown in Fig. 1.

In the sail reference frame the incident wave vector may be expressed

$$
\vec{k}_{i}^{\prime}=(2 \pi / \lambda)\left(-\sin \beta_{i, X} \hat{y}^{\prime}+\cos \beta_{i, X} \hat{z}^{\prime}\right)=(2 \pi / \lambda)\left(\sin \zeta_{X} \hat{y}^{\prime}+\cos \zeta_{X} \hat{z}^{\prime}\right)
$$

The diffracted wave vector $\vec{k}_{d}^{\prime}$ is determined from the phase matching condition, whereby the phase of the electric field tangential to the sail surface is continuous at the interface:

$$
\begin{aligned}
& \vec{k}_{i}^{\prime} \cdot \hat{x}^{\prime}=\vec{k}_{d}^{\prime} \cdot \hat{x}^{\prime}+m \vec{K} \cdot \hat{x}^{\prime} \\
& \vec{k}_{i}^{\prime} \cdot \hat{y}^{\prime}=\vec{k}_{d}^{\prime} \cdot \hat{y}^{\prime}+m \vec{K} \cdot \hat{y}^{\prime}
\end{aligned}
$$

where $m$ is the integer-valued diffraction order. For a normally incident beam where $\vec{k}_{i}^{\prime}$. $\left(\hat{x}^{\prime}+\hat{y}^{\prime}\right)=0$ and $\vec{k}_{d}^{\prime}=-m \vec{K}$, the beam is diffracted toward the sail axis as desired and discussed below when $m=-1$. 
Let us express the components of the diffracted wave vector by use of a unit vector $\hat{A}$ :

$$
\vec{k}_{d}^{\prime}=(2 \pi / \lambda)\left(A_{x^{\prime}} \hat{x}^{\prime}+A_{y^{\prime}} \hat{y}^{\prime}+A_{z^{\prime}} \hat{z}^{\prime}\right)
$$

where phase matching and elastic scattering $\left(\left|\vec{k}_{i}^{\prime}\right|=\mid \vec{k}_{d}^{\prime}\right) \mid$ provide

$$
\begin{aligned}
& A_{x^{\prime}}=-(m \lambda / \Lambda) \cos \psi \\
& A_{y^{\prime}}=-\sin \beta_{i, X}-(m \lambda / \Lambda) \sin \psi=\sin \zeta_{X}-(m \lambda / \Lambda) \sin \psi \\
& A_{z^{\prime}}= \pm\left(1-A_{x^{\prime}}^{2}-A_{y^{\prime}}^{2}\right)^{1 / 2}
\end{aligned}
$$

where the $-(+)$ sign corresponds to a reflection (transmission) grating. To achieve efficient acceleration along the beam axis we assume a reflection grating in this report.

Let us now describe diffraction in the stationary reference frame where $\vec{k}_{i}=(2 \pi / \lambda) \hat{Z}$ and

$$
\vec{k}_{d}=(2 \pi / \lambda)\left(B_{X} \hat{X}+B_{Y} \hat{Y}+B_{Z} \hat{Z}\right)
$$

where the unit vector $\hat{B}$ is the rotated version of $\hat{A}$ :

$$
\begin{aligned}
& B_{X}=A_{x^{\prime}} \\
& B_{Y}=A_{y^{\prime}} \cos \beta_{i, X}+A_{z^{\prime}} \sin \beta_{i, X}=A_{y^{\prime}} \cos \zeta_{X}-A_{z^{\prime}} \sin \zeta_{X} \\
& B_{Z}=-A_{y^{\prime}} \sin \beta_{i, X}+A_{z^{\prime}} \cos \beta_{i, X}=A_{y^{\prime}} \sin \zeta_{X}+A_{z^{\prime}} \cos \zeta_{X}
\end{aligned}
$$

General expressions relating rotated vectors $\hat{A}$ and $\hat{B}$ are described in "Appendix 2".

We therefore find the components of the efficiency vectors:

$$
\begin{array}{lrl}
\eta_{x^{\prime}}=(m \lambda / \Lambda) \cos \psi, & \eta_{X}=-B_{X} \\
\eta_{y^{\prime}}=(m \lambda / \Lambda) \sin \psi, & \eta_{Y}=-B_{Y} \\
\eta_{z^{\prime}}=\cos \beta_{i, X}-A_{z^{\prime}}=\cos \zeta_{X}-A_{z^{\prime}}, & \eta_{Z}=1-B_{Z}
\end{array}
$$

\section{Optomechanics of a diffractive sail}

The force and torque imparted to the sail produce both linear and angular displacements that depend on initial conditions and other factors such as the beam power, sail shape, and the spatial distribution of the grating vector. As depicted in Fig. 1 we assume a rigid circular sail of radius $a$ whose distribution in the sail reference frame $\mathscr{F}^{\prime}$ may be expressed:

$$
P_{\mathscr{F}^{\prime}}=\operatorname{Circ}\left(\sqrt{x^{\prime 2}+y^{\prime 2}} / a\right)
$$

where the function $\operatorname{Circ}(s)$ has a value of unity (zero) if $|s|<1(|s|>1)$. A payload of mass $M_{p}$ is attached to the sail of mass $M_{s}$ by means of a rigid boom of mass $M_{b}$ and length $D_{b}$ and negligible thickness. A positive (negative) value of $D_{b}$ corresponds to a non-exposed (exposed) payload. For convenience we assume $M_{s}=M_{p}$ such that the center of mass coincides with the mid-point of the boom. For this configuration the principal moment of inertia is $J_{x^{\prime}}=J_{y^{\prime}}=M_{s} a^{2} / 4+M_{s} D_{b}^{2} / 4+M_{p} D_{b}^{2} / 4$ and $J_{z^{\prime}}=M a^{2} / 2$ such that the sailcraft has a diagonal inertia tensor $J=\operatorname{diag}\left(J_{x^{\prime}}, J_{y^{\prime}}, J_{z^{\prime}}\right)$.

An observer standing next to a stationary laser system will observe the sail moving through space in the $\mathscr{F}=(X, Y, Z)$ coordinate system, where the reference frame $\mathscr{F}$ is described by a right-handed set of unit vectors $\{\hat{X}, \hat{Y}, \hat{Z}\}$ and origin $\mathscr{O}$. We wish to predict the position, velocity, and attitude of the sail in that inertial reference frame. However, radiation pressure 
exerted on the sail is more readily described in the non-inertial reference frame of the sail, $\mathscr{F}^{\prime}$, with right-handed coordinate system $\left(x^{\prime}, y^{\prime}, z^{\prime}\right)$ and origin $\mathscr{O}^{\prime}$ (see Fig. 1). In a homogeneous coordinate system (see "Appendix 3"), an arbitrary point in $\mathscr{F}\left(\mathscr{F}^{\prime}\right)$ is expressed as a column vector $[X, Y, Z, 1]^{T}\left(\left[x^{\prime}, y^{\prime}, z^{\prime}, 1\right]^{T}\right)$, where the 4 th component is a scaling factor set to unity.

Radiation pressure on a sail gives rise to forces and torques that may translate and rotate the sail. The translation of the sail in the frame $\mathscr{F}$ may be described by the displacement vector $\delta=\left[\delta_{X}, \delta_{Y}, \delta_{Z}\right]$. We represent the attitude of the sail in this frame in terms of ZYX sequence of Euler angles $\left\{\zeta_{Z}, \zeta_{Y}, \zeta_{X}\right\}$ (see "Appendix 1"). For an arbitrary rotation and translation the relationship between the two frames of reference may be expressed

$$
\left[\begin{array}{c}
x^{\prime} \\
y^{\prime} \\
z^{\prime} \\
1
\end{array}\right]=\mathbf{H}\left[\begin{array}{c}
X \\
Y \\
Z \\
1
\end{array}\right]=\left[\begin{array}{cccc}
c_{Y} c_{Z} & c_{Y} s_{Z} & -s_{Y} & \delta_{X} \\
s_{X} s_{Y} c_{Z}-c_{X} s_{Z} & s_{X} s_{Y} s_{Z}+c_{X} c_{Z} & s_{X} c_{Y} & \delta_{Y} \\
c_{X} s_{Y} c_{Z}+s_{X} s_{Z} & c_{X} s_{Y} s_{Z}-s_{X} c_{Z} & c_{X} c_{Y} & \delta_{Z} \\
0 & 0 & 0 & 1
\end{array}\right]\left[\begin{array}{c}
X \\
Y \\
Z \\
1
\end{array}\right]
$$

where $\mathbf{H}$ is the Homogeneous transformation matrix described in "Appendix 3", and the elements containing factors of $c_{X, Y, Z}=\cos \zeta_{X, Y, Z}$ and $s_{X, Y, Z}=\sin \zeta_{X, Y, Z}$ belong to the rotation matrix described in "Appendix 1".

The net radiation pressure force in the stationary reference frame is found by integrating over the local force elements:

$$
\vec{F}_{n e t}=(1 / c) \int_{-\infty}^{\infty} I P_{\mathscr{F}} \cos \phi \vec{\eta} d X d Y=M \ddot{\ddot{\delta}}
$$

where $P_{\mathscr{F}}$, is transformed into the reference frame $\mathscr{F}$ by the expression $P_{\mathscr{F}}=\mathbf{H}^{-1} P_{\mathscr{F}}$, $\phi$ is the angle between the sail normal and the incident wave vector (i.e., $\cos \phi=\hat{Z} \cdot \hat{z}^{\prime}$ ), $I=|E(X, Y, Z)|^{2}$ is the beam irradiance described in Eq. (1), $c$ is the speed of light, and we have applied Newton's second law to the right-hand side where $M=M_{s}+M_{p}+M_{b}$ is the total light sail mass.

Unlike the net force, the net torque $\vec{N}_{n e t}^{\prime}$ measured about the center of mass of the sail is calculated in the sail reference frame $\mathscr{F}^{\prime}$ and may be found by integration:

$$
\vec{N}_{n e t}^{\prime}=(1 / c) \int_{-\infty}^{\infty} \int_{\mathscr{F}^{\prime}} \cos \phi \vec{r}^{\prime} \times \vec{\eta}^{\prime} d x^{\prime} d y^{\prime}
$$

where $\vec{r}^{\prime}=x^{\prime} \hat{x}^{\prime}+y^{\prime} \hat{y}^{\prime}-\left(D_{b} / 2\right) \hat{z}^{\prime}$ is the moment arm. Euler's equations for rotational degrees of freedom may be expressed

$$
\begin{aligned}
& N_{n e t, x^{\prime}}=J_{x^{\prime}} \dot{\Omega}_{x^{\prime}}+\left(J_{z^{\prime}}-J_{y^{\prime}}\right) \Omega_{y^{\prime}} \Omega_{z^{\prime}} \\
& N_{n e t, y^{\prime}}=J_{y^{\prime}} \dot{\Omega}_{y^{\prime}}+\left(J_{x^{\prime}}-J_{z^{\prime}}\right) \Omega_{z^{\prime}} \Omega_{x^{\prime}} \\
& N_{n e t, z^{\prime}}=J_{z^{\prime}} \dot{\Omega}_{z^{\prime}}+\left(J_{y^{\prime}}-J_{x^{\prime}}\right) \Omega_{x^{\prime}} \Omega_{y^{\prime}}
\end{aligned}
$$

where the angular velocity of the sail measured in the reference frame $\mathscr{F}^{\prime}$ is related to the time rate of change of Euler angles (see "Appendix 1").

$$
\dot{\vec{\Omega}}^{\prime}=\left(\dot{\zeta}_{X}-s_{X} \dot{\zeta}_{Z}\right) \hat{x}^{\prime}+\left(c_{X} \dot{\zeta}_{Y}+c_{Y} s_{X} \dot{\zeta}_{Z}\right) \hat{y}^{\prime}+\left(-s_{X} \dot{\zeta}_{Y}+c_{Y} c_{X} \dot{\zeta}_{Z}\right) \hat{z}^{\prime}
$$

and where the dot symbol represents the time derivative. The displacement, velocity, attitude, and angular velocity of the sail may be found by simultaneously solving coupled equations, Eqs. (13)-(16). 


\section{Linear stability analysis of a diffractive sail}

From a practical point of view we desire the sail to accelerate in the $\hat{Z}$ direction, while otherwise at an equilibrium position centered on the beam and an equilibrium attitude with the sail axis parallel to the optical axis. To determine whether a given set of system parameters satisfies this requirement, linear stability analysis is applied [50]. Let us define a state vector: $\mathbf{q}=\left[\delta_{X}, \delta_{Y}, \zeta_{X}, \zeta_{Y}, \dot{\delta}_{X}, \dot{\delta}_{Y}, \dot{\Omega}_{X}, \dot{\Omega}_{Y}\right]^{T}$. The linearized equations of motion for translation and rotation may be expressed:

$$
\dot{\mathbf{q}}=\boldsymbol{\Gamma}_{0} \mathbf{q}=\left[\begin{array}{c}
\dot{\delta}_{X} \\
\dot{\delta}_{Y} \\
\dot{\Omega}_{X} \\
\dot{\Omega}_{Y} \\
\ddot{\delta}_{X} \\
\ddot{\delta}_{Y} \\
\ddot{\Omega}_{X} \\
\ddot{\Omega}_{Y}
\end{array}\right]=\left[\begin{array}{cccccccc}
0 & 0 & 0 & 0 & 1 & 0 & 0 & 0 \\
0 & 0 & 0 & 0 & 0 & 1 & 0 & 0 \\
0 & 0 & 0 & 0 & 0 & 0 & 1 & 0 \\
0 & 0 & 0 & 0 & 0 & 0 & 0 & 1 \\
\Gamma_{1} & \Gamma_{2} & 0 & 0 & 0 & 0 & 0 & 0 \\
\Gamma_{3} & \Gamma_{4} & 0 & 0 & 0 & 0 & 0 & 0 \\
0 & 0 & \Gamma_{5} & \Gamma_{6} & 0 & 0 & 0 & 0 \\
0 & 0 & \Gamma_{7} & \Gamma_{8} & 0 & 0 & 0 & 0
\end{array}\right]_{\mathbf{q}_{0}}\left[\begin{array}{c}
\delta_{X} \\
\delta_{Y} \\
\zeta_{X} \\
\zeta_{Y} \\
\dot{\delta}_{X} \\
\dot{\delta}_{Y} \\
\dot{\Omega}_{X} \\
\dot{\Omega}_{Y}
\end{array}\right]
$$

where $\boldsymbol{\Gamma}_{\mathbf{0}}$ is calculated at the equilibrium state $\mathbf{q}_{\mathbf{0}}=\mathbf{0}$ :

$$
\begin{aligned}
& \Gamma_{1}=\left.\frac{1}{M} \frac{\partial\left(F_{X} / F_{0}\right)}{\partial \delta_{X}}\right|_{\mathbf{q}_{0}}, \Gamma_{2}=\left.\frac{1}{M} \frac{\partial\left(F_{X} / F_{0}\right)}{\partial \zeta_{Y}}\right|_{\mathbf{q}_{0}}, \Gamma_{3}=\left.\frac{1}{J_{y^{\prime}}} \frac{\partial\left(N_{y^{\prime}} / N_{0}\right)}{\partial \delta_{X}}\right|_{\mathbf{q}_{0}}, \\
& \Gamma_{4}=\left.\frac{1}{J_{y^{\prime}}} \frac{\partial\left(N_{y^{\prime}} / N_{0}\right)}{\partial \zeta_{Y}}\right|_{\mathbf{q}_{0}}, \\
& \Gamma_{5}=\left.\frac{1}{M} \frac{\partial\left(F_{Y} / F_{0}\right)}{\partial \delta_{Y}}\right|_{\mathbf{q}_{0}}, \Gamma_{6}=\left.\frac{1}{M} \frac{\partial\left(F_{Y} / F_{0}\right)}{\partial \zeta_{X}}\right|_{\mathbf{q}_{0}}, \Gamma_{7}=\left.\frac{1}{J_{x^{\prime}}} \frac{\partial\left(N_{x^{\prime}} / N_{0}\right)}{\partial \delta_{Y}}\right|_{\mathbf{q}_{0}}, \\
& \Gamma_{8}=\left.\frac{1}{J_{x^{\prime}}} \frac{\partial\left(N_{x^{\prime}} / N_{0}\right)}{\partial \zeta_{X}}\right|_{\mathbf{q}_{0}}
\end{aligned}
$$

By calculating the eigenvalues of the Jacobian of $\boldsymbol{\Gamma}_{0}$, we determine complex frequencies that correspond to state solutions having the time-dependent form $\exp \left(\gamma_{a}, b t\right)$, where real values of $\gamma_{a, b}$ provide exponential damping or gain, and imaginary values provide oscillations. Four complex frequencies are found which satisfy:

$$
\begin{aligned}
& \gamma_{a}= \pm \sqrt{\frac{1}{2}\left(\Gamma_{1}+\Gamma_{4} \pm \sqrt{\left(\Gamma_{1}-\Gamma_{4}\right)^{2}+4 \Gamma_{2} \Gamma_{3}}\right)} \equiv \gamma_{a, r}+i \omega_{a} \\
& \gamma_{b}= \pm \sqrt{\frac{1}{2}\left(\Gamma_{5}+\Gamma_{8} \pm \sqrt{\left(\Gamma_{5}-\Gamma_{8}\right)^{2}+4 \Gamma_{6} \Gamma_{7}}\right)} \equiv \gamma_{b, r}+i \omega_{b}
\end{aligned}
$$

where $\gamma_{a, r}, \gamma_{b, r}, \omega_{a}, \omega_{b}$ are real values. The conditions for linear stability are $\gamma_{a, r} \leq 0$ and $\gamma_{b, r} \leq 0$, i.e., exponential growth is prohibited. For $\gamma_{a, r}=\gamma_{b, r}=0$ as found below, the sail oscillates about the equilibrium point with four characteristic periods that depend on system parameters such as the grating period, the size of the sail, the beam size and power, and the moment of inertia of the light sail. What is more, for the symmetric system considered in this report $\Gamma_{1}=\Gamma_{5}<0, \Gamma_{4}=\Gamma_{8}=0$, and $\Gamma_{2} \Gamma_{3}=\Gamma_{6} \Gamma_{7}<0, \Gamma_{1}^{2}>4\left|\Gamma_{2} \Gamma_{3}\right|$ and we therefore find two degenerate frequencies: a high frequency $\omega_{h}$ and a low frequency $\omega_{l}$ satisfying

$$
\begin{aligned}
& \omega_{h}^{2}=(1 / 2)\left(\omega_{0}^{2}+\Delta^{2}\right) \\
& \omega_{l}^{2}=(1 / 2)\left(\omega_{0}^{2}-\Delta^{2}\right)
\end{aligned}
$$


Table 1 List of parameters and values

\begin{tabular}{|c|c|}
\hline Parameters & Value \\
\hline \multicolumn{2}{|l|}{ Light Sail } \\
\hline Grating period, $\Lambda$ & $1.6 \mu \mathrm{m}$ \\
\hline Diffraction order, $m$ & -1 \\
\hline Radius, $a$ & $1.0 \mathrm{~m}$ \\
\hline Mass, $M_{S}$ & $0.50 \mathrm{~g}$ \\
\hline Payload mass, $M_{p}$ & $0.50 \mathrm{~g}$ \\
\hline Boom length, $D_{b}$ & $15.0 \mathrm{~m}$ \\
\hline Boom mass, $M_{b}$ & $0.17 \mathrm{~g}$ \\
\hline Total mass, $M$ & $1.17 \mathrm{~g}$ \\
\hline Moments of inertia, $J_{x}^{\prime}, J_{y}^{\prime}$ & $0.06 \mathrm{~kg} \mathrm{~m}^{-2}$ \\
\hline Moments of inertia, $J_{z}^{\prime}$ & $0.25 \mathrm{~g} \mathrm{~m}^{-2}$ \\
\hline Radius of gyration, $R_{g}$ & $7.13 \mathrm{~m}$ \\
\hline \multicolumn{2}{|l|}{ Laser beam } \\
\hline Power, $P_{0}$ & $10 \mathrm{~kW}$ \\
\hline Gaussian beam waist, $w_{0}$ & $0.5 \mathrm{~m}$ \\
\hline Wavelength, $\lambda$ & $1.0 \mu \mathrm{m}$ \\
\hline Diffraction length, $Z_{0}$ & $0.79 \times 10^{6} \mathrm{~m}$ \\
\hline \multicolumn{2}{|l|}{ System } \\
\hline$\Gamma_{1}=\Gamma_{5}$ & $-0.04 \mathrm{~kg}^{-1} \mathrm{~m}^{-1}$ \\
\hline$\Gamma_{2}=\Gamma_{6}$ & $0.05 \mathrm{~kg}^{-1} \mathrm{rad}^{-1}$ \\
\hline$\Gamma_{3}=\Gamma_{7}$ & $-0.005 \mathrm{~kg}^{-1} \mathrm{~m}^{-3}$ \\
\hline$\Gamma_{4}=\Gamma_{8}$ & $0 \mathrm{~kg}^{-1} \mathrm{~m}^{-2} \mathrm{rad}^{-1}$ \\
\hline$\omega_{0}$ & $0.2 \mathrm{rad} / \mathrm{s}$ \\
\hline$\Delta$ & $0.157 \mathrm{rad} / \mathrm{s}$ \\
\hline$\omega_{l}$ & $0.087 \mathrm{rad} / \mathrm{s}$ \\
\hline$\omega_{h}$ & $0.18 \mathrm{rad} / \mathrm{s}$ \\
\hline$T_{h}$ & $35 \mathrm{~s}$ \\
\hline$T_{l}$ & $72 \mathrm{~s}$ \\
\hline \multicolumn{2}{|l|}{ Initial conditions $(t=0)$ : } \\
\hline Displacement, $\left(\delta_{X}, \delta_{y}\right)$ & $(0.1 \mathrm{~m},-0.1 \mathrm{~m})$ \\
\hline Attitude, $\left(\zeta_{X}, \zeta_{Y}\right)$ & $\left(1^{\circ},-1^{\circ}\right)$ \\
\hline Linear velocity, $\left(\dot{\delta}_{X}, \dot{\delta}_{Y}\right)$ & $(0,0)$ \\
\hline Angular velocity, $\left(\dot{\Omega}_{X}, \dot{\Omega}_{Y}\right)$ & $(0,0)$ \\
\hline
\end{tabular}

where $\omega_{0}^{2}=\Gamma_{1}+\Gamma_{4}=\Gamma_{5}+\Gamma_{8}$ and $\Delta^{2}=\left(\left(\Gamma_{1}-\Gamma_{4}\right)^{2}+4 \Gamma_{2} \Gamma_{3}\right)^{1 / 2}=\left(\left(\Gamma_{5}-\Gamma_{8}\right)^{2}+\right.$ $\left.4 \Gamma_{6} \Gamma_{7}\right)^{1 / 2}$. Therefore we expect the system to display two oscillation modes when excited close to equilibrium.

\section{Numerical solutions}

Closed-form solutions of the system equations of motion generally do not exist, and therefore, numerical integration methods must be applied. For a representative non-optimized case we examined a laser-sail system having parameters listed in Table 1 . We assumed a beam power 

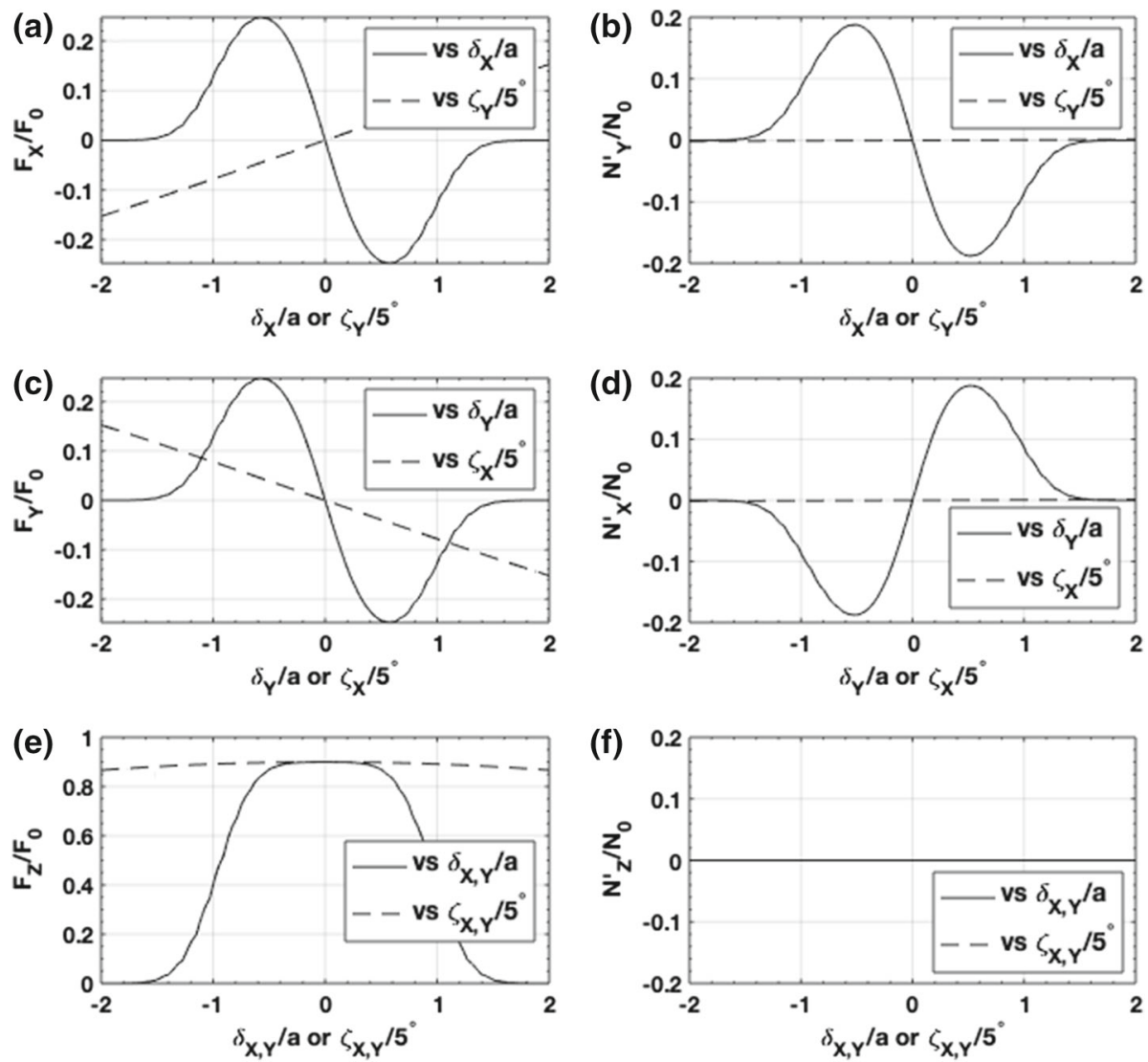

Fig. 2 Normalized components of $\mathbf{a}, \mathbf{c}, \mathbf{e}$ force and $\mathbf{b}, \mathbf{d}, \mathbf{f}$ torque as a function of transverse displacement, $\delta_{X, Y}$, and attitude, $\zeta_{X, Y}$, where $F_{0}=2 P_{0} / c$ and $N_{0}=F_{0} D_{b} / 2$

of $P_{0}=10[\mathrm{~kW}]$ (as was used in microwave beam-rider experiments $[17,18]$ ) illuminating a sail of radius $a=1[\mathrm{~m}]$, with the beam waist $w_{0}=0.5[\mathrm{~m}]$ under-filling the sail.

We numerically computed Eqs. (13)-(16) for different initial values of linear and angular displacement, plotting the results in Fig. 2. The linear nature of the force and torque nearequilibrium is clearly evident in Fig. 2 for the range $\left|\delta_{X, Y} / a\right|<0.5$ and $\left|\zeta_{X, Y}\right|<2.5^{\circ}$. We also observe that the force along the beam axis reaches roughly $90 \%$ of the maximum theoretical value of $2 P_{0} / c$. Furthermore, the value of the roll torque $N_{z}^{\prime}$ is zero, and thus the system does not acquire angular momentum about the sail axis. Changing only the angle $\zeta_{X}$ $\left(\zeta_{Y}\right)$ at equilibrium we also find that the torque $N_{Y}^{\prime}\left(N_{X}^{\prime}\right)$ is zero valued.

A perspective of the net force exerted on the sail at equilibrium is depicted in Fig. 3a where local transverse components of force are displayed, resulting in no net transverse force. Similarly, the net torque exerted on the sail at equilibrium is depicted in Fig. $3 \mathrm{~b}$ where local transverse components of torque, are displayed, resulting in no net transverse torque. If the sail is displaced from equilibrium to the right, as in Fig. $3 \mathrm{c}$ the net force drives the sail to the left. In Fig. $3 \mathrm{~d}$ the net torque is in the $-\hat{Y}$ direction.

Values of the slopes at the equilibrium points are obtained from Fig. 2, which along with the mass and moments of inertia in Table 1 allow us to determine the values of $\Gamma_{j}$ (see 


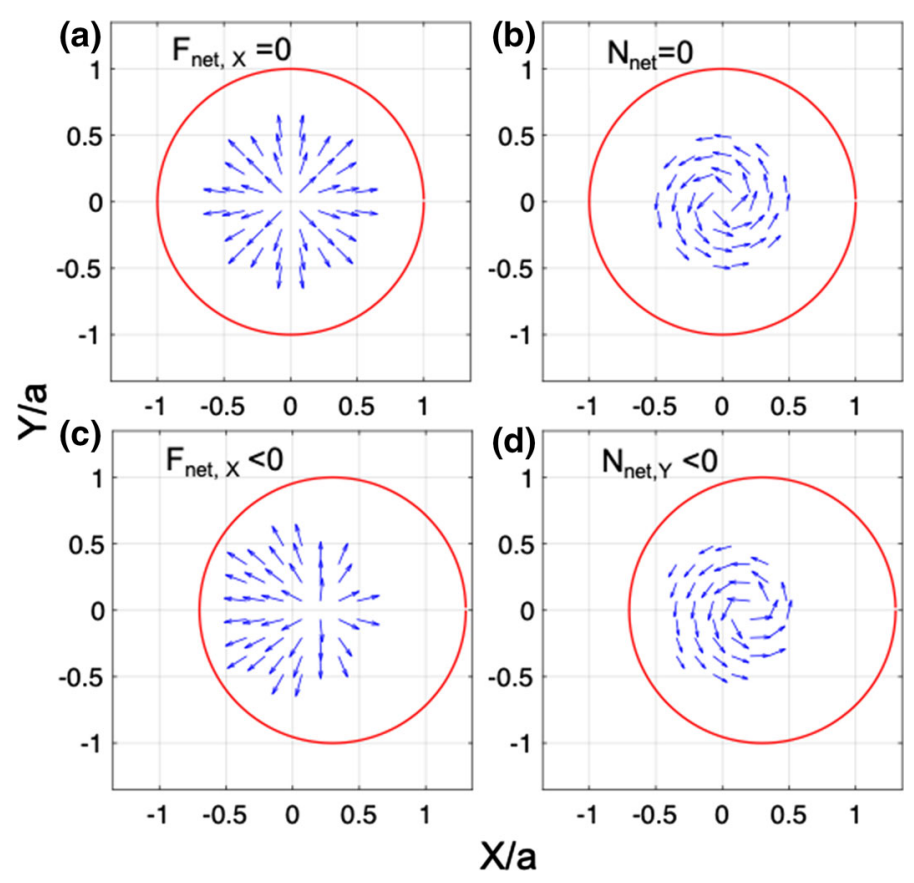

Fig. 3 Local forces and torques exerted on an non-tilted axicon sail when the sail and optical axis are a, b co-linear, and $\mathbf{c}, \mathbf{d}$ displaced. $\mathbf{c}$ The net force drives the sail toward the beam axis $(X, Y)=(0,0)$

Eq. (18)). Inserting $\Gamma_{j}$ into Eq. (20) we find $\omega_{h}=0.18[\mathrm{rad} / \mathrm{s}]$ and $\omega_{l}=0.087[\mathrm{rad} / \mathrm{s}]$, with respective oscillation periods $T_{h}=35[\mathrm{~s}]$ and $T_{l}=72[\mathrm{~s}]$. For a higher power laser beam $\tilde{P}$ we expect proportionally more optomechanical energy to be pumped into the system [28], resulting in higher squared values of frequency and lower squared values of the oscillation periods, $\tilde{T}_{h, l}$. Therefore

$$
\tilde{T}_{h, l}=\left(P_{0} / \tilde{P}\right)^{1 / 2} T_{h, l}
$$

For example, if $P=1[\mathrm{GW}]$ the periods are expected to decrease to $T_{h}=11[\mathrm{~ms}]$ and $T_{l}=228[\mathrm{~ms}]$.

Solutions of the equations of motion for a given set of initial conditions were numerically solved by use of the fourth-order Runge-Kutta method. An example that illustrates stable motion for small perturbations from equilibrium is shown in Fig. 4 for the system initially at rest and displaced: $\delta_{X} / a=-\delta_{Y} / a=0.1$ and $\zeta_{X}=-\zeta_{Y}=1^{\circ}$. The phase diagrams correspond to an elapsed time of $t=780 T_{h}=27,400[\mathrm{~s}]$. During this time the sail acquires a speed of $\Delta v_{Z}=1.4[\mathrm{~km} / \mathrm{s}]$ and traverses a distance of $\Delta Z=19 \times 10^{6}[\mathrm{~km}]=25 Z_{0}$, assuming the beam size is controlled, so that it does not overfill the sail. As expected from our linear stability analysis, the system remains stable under this condition. The acceleration $a_{Z}=0.51\left[\mathrm{~m} / \mathrm{s}^{2}\right]$ may be increased in proportion to the laser power, thereby providing values of $\Delta v_{Z}$ that are relevant for orbit-changing maneuvers, although the high oscillation frequencies (see above) may become mechanically intolerable if not damped.

An examination of Fig. 5 indicates that force and torque are nonlinearly related to linear and angular displacements for $\left|\delta_{X, Y} / a\right| \gtrsim 0.5$ and $\left|\zeta_{X, Y}\right| \gtrsim 2.5^{\circ}$. Below these bounds the system may be characterized by linear and torsional spring models with stiffness values equal to the slopes in Fig. 2. Close to the nonlinear bounds the springs become soft and less able 

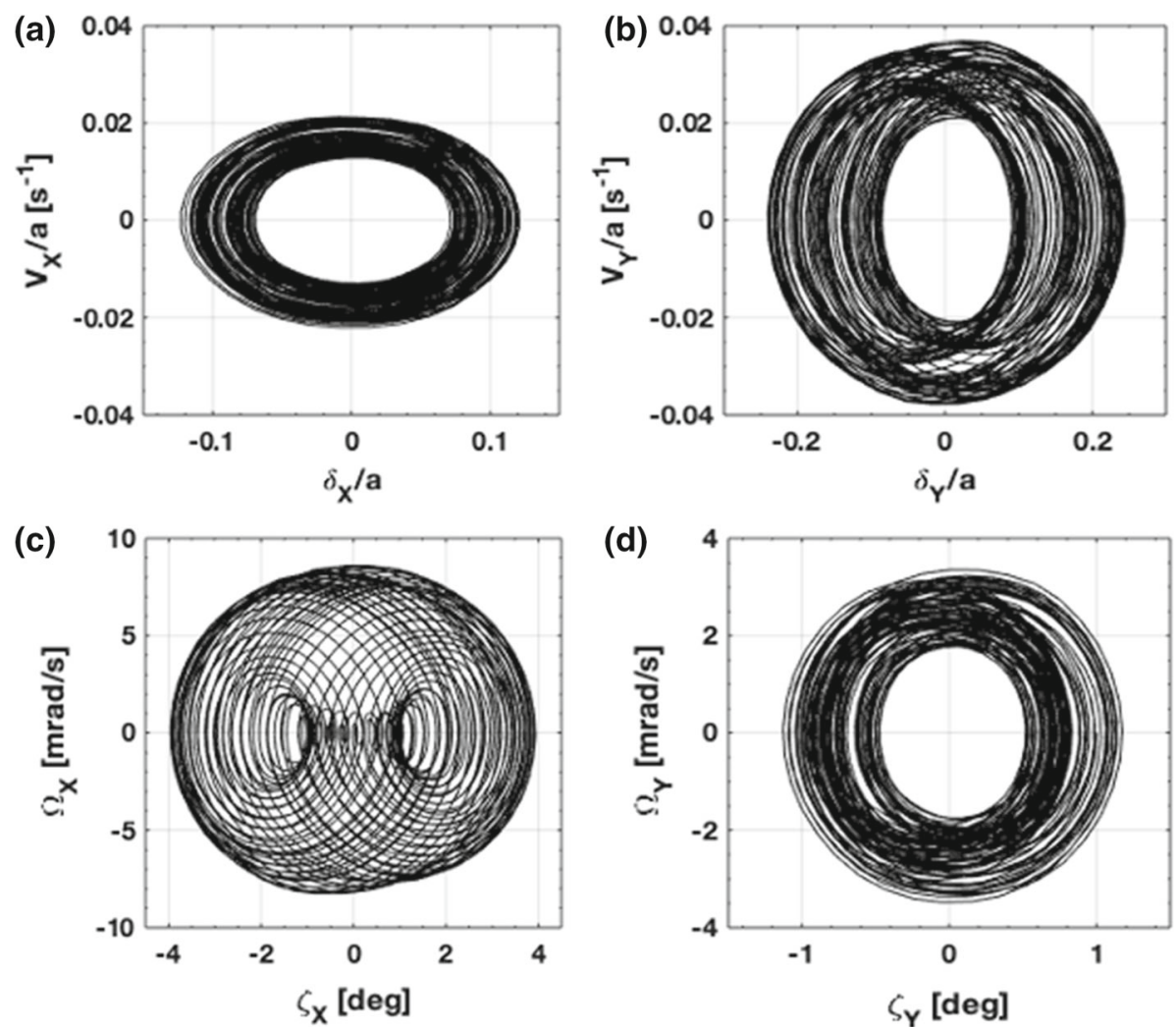

Fig. 4 Phase plot for oscillations $\mathbf{a}, \mathbf{b}$ along transverse direction and $\mathbf{c}, \mathbf{d}$ in attitude about $(\hat{X}, \hat{Y})$ is shown, for an initial condition $\boldsymbol{q}=\left[0.1,-0.1,1^{\circ},-1^{\circ}, 0,0,0,0\right]^{T}$ and time $t=27,400[\mathrm{~s}]$

to provide a restoring force or torque. Beyond these bounds the system is driven away from equilibrium. To explore how the departure from linear behavior affects the range of stable motion for the system described in Table 1 we varied the initial conditions across the range $\delta_{X, Y} \in[-a, a]$, or $\zeta_{X, Y} \in\left[-10^{\circ}, 10^{\circ}\right]$, with $\dot{\delta}_{X, Y}=\dot{\Omega}_{X, Y}=0$. We then numerically integrated the coupled equations of motion, categorized the observed motion as stable or unstable, and summarized the results in the stability maps shown in Fig. 5. The stable range of linear displacement (assuming $\zeta_{X, Y}=\dot{\delta}_{X, Y}=\dot{\Omega}_{X, Y}=0$ at $t=0$ indicates a stability zone defined by $\delta_{X}^{2}+\delta_{Y}^{2} \leq(0.3 a)^{2}$ where the radius $0.3 a$ is significantly smaller than the bound $\delta_{X, Y}=0.5 a$. We attribute this smaller zone to the weak force stiffness at $0.3 a$ and coupling to motion in other degrees of freedom that do not provide an attraction to equilibrium. A linear zone boundary was found when varying both $\delta_{X}$ and $\zeta_{Y}$ (with other state parameters equal to zero), and is shown in Fig. 5b. An examination of Fig. $5 \mathrm{~b}$ indicates that the force at $\zeta_{Y}=6^{\circ}$ is equal and opposite to the force at $\delta_{X}=0.3 a$, suggesting both a reason and an equivalence for the stability boundaries at $\delta_{X}=0.3 a$ and $\zeta_{Y}=6^{\circ}$. The same zone boundary relation was found when varying $\delta_{Y}$ and $\zeta_{X}$. According to Fig. $5 \mathrm{c}$ the system stability is more robust to simultaneous displacements along and rotations about a common axis. Finally, we explore an example where variations of the boom length and beam size affect stability. In this example, we selected the initial condition: $\delta_{X}=-\delta_{Y}=0.1 a$ and $\zeta_{X}=-\zeta_{Y}=1^{\circ}$. As shown in Fig. 5d the system is generally more stable for long boom lengths, but for a given 

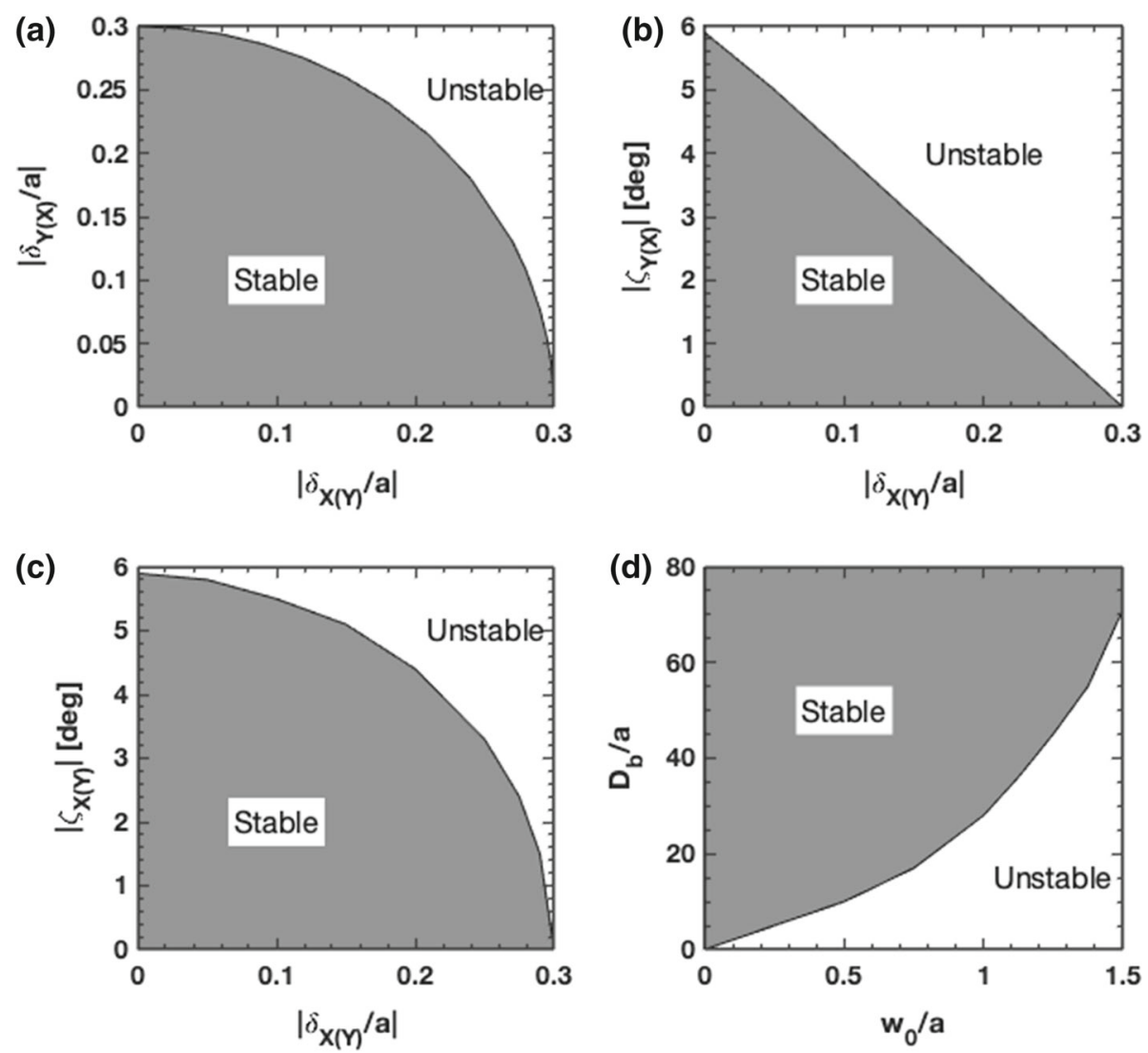

Fig. 5 Regions of optomechanical stability for a relative linear displacement $\delta_{X} / a$ versus $\delta_{Y} / a$, b orthogonal linear displacement and attitude axes, $\delta_{X} / a$ versus $\zeta_{Y}$ (or $\delta_{Y} / a$ versus $\zeta_{X}$ ), c parallel linear displacement and attitude axes, $\delta_{X} / a$ versus $\zeta_{X}$ (or $\delta_{Y}$ versus $\zeta_{Y}$ ) and $\mathbf{d}$ relative laser beam width versus relative boom length $w_{0} / a$ versus $D_{b} / a$

beam size there is a minimum boom length below which the system is unstable. For example, if the beam radius equals half the sail radius, $w_{0}=a / 2$, as listed in Table 1 , we predict a minimum boom length of $D_{b}=10 a$. In comparison we made our numerical studies in Section 3 for a boom length of $D_{b}=15 a$, well into the stable regime. We also predict that stable motion may be achieved when the beam overfills the sail (i.e., $w_{0}>a$ ), but only if the boom length is made significantly larger than the sail radius. For example if $w_{0}=a$, stability requires $D_{b}>28 a$.

\section{Summary}

Diffraction-based light sails provide a design flexibility that is not afforded by reflective sails. This is attributed to the controlled redirection of light by an engineered diffractive surface rather than a deformed reflective surface. We have described the optomechanics of a rigid non-spinning laser-driven sail comprised of a reflective axicon diffraction grating and a payload attached to a boom. A single diffraction order is assumed, producing diffraction toward 
the optical axis. Such diffraction affords passive stability while also providing longitudinal acceleration along the beam axis. Our example exhibited $90 \%$ of the maximum theoretical value of force along the optical axis, with the $10 \%$ deficit sacrificed to achieve beam-riding stability against transverse perturbations as large as $30 \%$ of the sail radius and attitude perturbations as large as $6^{\circ}$. Numerical methods were used to integrate the coupled equations of motion, allowing us to illustrate stable oscillations and to map regions of stable and unstable motion. A linear stability analysis predicted as many as four modes of oscillation, reducing to two degenerate frequencies for our symmetric structure. Both the attitude and transverse motion exhibited the two frequencies. The squared frequency was found to increase linearly with beam power. Our optomechanical model may be readily extended to include complex diffractive structures, complex beam shapes, modulated beam power, and a spinning sail. The model requires further work to include the Doppler effect [51], mechanical compliance, and center-of-mass center-of-pressure offsets. Advanced features that may be integrated into the diffractive sail include active attitude control $[31,45,52]$.

Acknowledgements The authors acknowledge funding and support from National Aeronautics and Space Administration (NASA, Innovative Advanced Concepts Office), Award Number 80NSSC18K0867. We are grateful to Ying-Ju Lucy Chu and Amber Dubill, Rochester Institute of Technology, for discussions related to radiation pressure and rigid body dynamics, and to Les Johnson and Andy Heaton, NASA Marshall Space Flight Center, for discussions on light sailing.

Open Access This article is licensed under a Creative Commons Attribution 4.0 International License, which permits use, sharing, adaptation, distribution and reproduction in any medium or format, as long as you give appropriate credit to the original author(s) and the source, provide a link to the Creative Commons licence, and indicate if changes were made. The images or other third party material in this article are included in the article's Creative Commons licence, unless indicated otherwise in a credit line to the material. If material is not included in the article's Creative Commons licence and your intended use is not permitted by statutory regulation or exceeds the permitted use, you will need to obtain permission directly from the copyright holder. To view a copy of this licence, visit http://creativecommons.org/licenses/by/4.0/.

\section{Appendix 1: Euler angles}

Let $\mathscr{F}=\{\hat{X}, \hat{Y}, \hat{Z}\}$ represent the reference frame of a stationary coordinate system and $\mathscr{F}^{\prime}=$ $\{\hat{x}, \hat{y}, \hat{z}\}$ represent a rotated reference frame. The two frames of reference with orthonormal coordinate systems can be aligned by three consecutive rotations given by Euler angles $\zeta \in\left\{\zeta_{Z}, \zeta_{Y}, \zeta_{X}\right\}$. As per Euler theorem, two consecutive rotations have to be made about different axis. We make use of the $Z Y X$ Euler angle sequence with rotation matrices:

$$
\xi_{Z}=\left(\begin{array}{ccc}
c_{Z} & s_{Z} & 0 \\
-s_{Z} & c_{Z} & 0 \\
0 & 0 & 0
\end{array}\right), \quad \xi_{Y}=\left(\begin{array}{ccc}
c_{Y} & 0 & -s_{Y} \\
0 & 1 & 0 \\
s_{Y} & 0 & c_{Y}
\end{array}\right), \quad \xi_{X}=\left(\begin{array}{ccc}
1 & 0 & 0 \\
0 & c_{X} & s_{X} \\
0 & -s_{X} & c_{X}
\end{array}\right)
$$

where $\xi_{j}$ is the rotation about $\mathrm{j}$-th axis by angle $\zeta_{j}$ and $c_{j}=\cos \zeta_{j}$ and $s_{j}=\sin \zeta_{j}$, and $j=X, Y$, or $Z$, with the singularity restriction $\zeta_{Y} \in(-\pi / 2, \pi / 2)$ and $\zeta_{X, Z} \in(0,2 \pi)$. The rotated frame may be expressed $\mathscr{F}^{\prime}=\boldsymbol{\xi} \mathscr{F}$ where

$$
\boldsymbol{\xi}=\xi_{X} \xi_{Y} \xi_{Z}=\left[\begin{array}{ccc}
c_{Y} c_{Z} & c_{Y} s_{Z} & -s_{Y} \\
s_{X} s_{Y} c_{Z}-c_{X} s_{Z} & s_{X} s_{Y} s_{Z}+c_{X} c_{Z} & s_{X} c_{Y} \\
c_{X} s_{Y} c_{Z}+s_{X} s_{Z} & c_{X} s_{Y} s_{Z}-s_{X} c_{Z} & c_{X} c_{Y}
\end{array}\right]
$$


Conversely we may write $\mathscr{F}=\xi^{-1} \mathscr{F}^{\prime}$ where

$$
\boldsymbol{\xi}^{-1}=\boldsymbol{\xi}^{T}=\left[\begin{array}{ccc}
c_{Y} c_{Z} & s_{X} s_{Y} c_{Z}-c_{X} s_{Z} & c_{X} s_{Y} c_{Z}+s_{X} s_{Z} \\
c_{Y} s_{Z} & s_{X} s_{Y} s_{Z}+c_{X} c_{Z} & c_{X} s_{Y} s_{Z}-s_{X} c_{Z} \\
-s_{Y} & s_{X} c_{Y} & c_{X} c_{Y}
\end{array}\right]
$$

The angular velocity $\Omega$ depends on the time rate of change of Euler angles $\dot{\zeta}=$ $\left[\dot{\zeta}_{X}, \dot{\zeta}_{Y}, \dot{\zeta}_{Z}\right]^{T}$ where $\dot{\zeta}_{j}=d \zeta_{j} / d t$. For the $Z Y X$ sequence of Euler angles, the angular velocity in the two frames may be expressed [53]:

$$
\boldsymbol{\Omega}^{\prime}=\boldsymbol{\Omega}=\left(\begin{array}{ccc}
1 & 0 & -s_{X} \\
0 & c_{X} & c_{Y} s_{X} \\
0 & -s_{X} & c_{Y} c_{X}
\end{array}\right)\left(\begin{array}{l}
\dot{\zeta}_{X} \\
\dot{\zeta}_{Y} \\
\dot{\zeta}_{Z}
\end{array}\right)
$$

and the angular velocity vectors are expressed

$$
\begin{aligned}
\overrightarrow{\mathbf{\Omega}^{\prime}} & =\left(\zeta_{X}-s_{X} \zeta_{Z}\right) \hat{x^{\prime}}+\left(c_{X} \zeta_{Y}+c_{Y} s_{X} \zeta_{Z}\right) \hat{y^{\prime}}+\left(-s_{X} \zeta_{Y}+c_{Y} c_{X} \zeta_{Z}\right) \hat{z^{\prime}} \\
\overrightarrow{\boldsymbol{\Omega}} & =\left(\zeta_{X}-s_{X} \zeta_{Z}\right) \hat{X}+\left(c_{X} \zeta_{Y}+c_{Y} s_{X} \zeta_{Z}\right) \hat{Y}+\left(-s_{X} \zeta_{Y}+c_{Y} c_{X} \zeta_{Z}\right) \hat{Z}
\end{aligned}
$$

\section{Appendix 2: Rotation of diffraction efficiency vectors}

For an arbitrarily orientated sail with respect to a stationary frame, the incident beam, grating vector, and diffracted beam are expressed:

$$
\begin{aligned}
\vec{k}_{i} & =(2 \pi / \lambda) \hat{Z} \text { and } \vec{k}_{i}^{\prime}=(2 \pi / \lambda)\left(-s_{Y} \hat{x}^{\prime}+s_{X} c_{Y} \hat{y}^{\prime}+c_{X} c_{Y} \hat{z}^{\prime}\right) \\
\vec{K} & =-(2 \pi / \Lambda)\left(\cos \psi \hat{x}^{\prime}+\sin \psi \hat{y}^{\prime}\right) \\
\overrightarrow{k_{d}^{\prime}} & =(2 \pi / \lambda)\left(A_{x^{\prime}} \hat{x}^{\prime}+A_{y^{\prime}} \hat{y}^{\prime}+A_{z^{\prime}} \hat{z}^{\prime}\right)
\end{aligned}
$$

where

$$
\begin{aligned}
& A_{x^{\prime}}=k_{i, x^{\prime}}-m K_{x^{\prime}}=-s_{Y}-(m \lambda / \Lambda) \cos \psi \\
& A_{y^{\prime}}=k_{i, y^{\prime}}-m K_{y^{\prime}}=s_{X} c_{Y}-(m \lambda / \Lambda) \sin \psi \\
& A_{z^{\prime}}=k_{d, z^{\prime}}= \pm \sqrt{1-A_{x^{\prime}}^{2}-A_{y^{\prime}}^{2}}
\end{aligned}
$$

In the stationary frame, the diffracted beam components are expressed

$$
\vec{k}_{d}=(2 \pi / \lambda)\left(B_{X} \hat{X}+B_{Y} \hat{Y}+B_{Z} \hat{Z}\right)
$$

where

$$
\left[\begin{array}{c}
B_{X} \\
B_{Y} \\
B_{Z}
\end{array}\right]=\left[\begin{array}{ccc}
c_{Y} c_{Z} & s_{X} s_{Y} c_{Z}-c_{X} s_{Z} & c_{X} s_{Y} c_{Z}+s_{X} s_{Z} \\
c_{Y} s_{Z} & s_{X} s_{Y} s_{Z}+c_{X} c_{Z} & c_{X} s_{Y} s_{Z}-s_{X} c_{Z} \\
-s_{Y} & s_{X} c_{Y} & c_{X} c_{Y}
\end{array}\right]\left[\begin{array}{c}
A_{x^{\prime}} \\
A_{y^{\prime}} \\
A_{z^{\prime}}
\end{array}\right]=\xi^{T}\left[\begin{array}{c}
A_{x^{\prime}} \\
A_{y^{\prime}} \\
A_{z^{\prime}}
\end{array}\right]
$$

The photon momentum transfer efficiency imparted to the sail may be expressed

$$
\vec{\eta}^{\prime}=\left(\vec{k}_{i}^{\prime}-\vec{k}_{d}^{\prime}\right) /(2 \pi / \lambda) \text { and } \vec{\eta}=\left(\vec{k}_{i}-\vec{k}_{d}\right) /(2 \pi / \lambda)
$$


or

$$
\begin{aligned}
& \eta_{x^{\prime}}=\left(\vec{k}_{\mathrm{i}}^{\prime}-\vec{k}_{d}^{\prime}\right) /(2 \pi / \lambda)=\left(-s_{Y}-A_{x^{\prime}}\right) \\
& \eta_{y^{\prime}}=\left(\vec{k}_{i}^{\prime}-\vec{k}_{d}^{\prime}\right) /(2 \pi / \lambda)=\left(s_{X} c_{Y}-A_{y^{\prime}}\right) \\
& \eta_{z^{\prime}}=\left(\vec{k}_{i}^{\prime}-\vec{k}_{d}^{\prime}\right) /(2 \pi / \lambda)=\left(c_{X} c_{Y}-A_{z^{\prime}}\right)
\end{aligned}
$$

and

$$
\begin{gathered}
\eta_{x}=\left(\vec{k}_{i}-\vec{k}_{d}\right) /(2 \pi / \lambda)=-B_{X} \\
\eta_{y}=\left(\vec{k}_{i}-\vec{k}_{d}\right) /(2 \pi / \lambda)=-B_{Y} \\
\eta_{z}=\left(\vec{k}_{i}-\vec{k}_{d}\right) /(2 \pi / \lambda)=1-B_{Z}
\end{gathered}
$$

\section{Appendix 3: Homogeneous coordinates}

Let $[X, Y, Z]^{T}$ be the Cartesian coordinates of a point in a reference frame $\mathscr{F}$ with origin $\mathscr{O}$ and a right-handed basis $\{\hat{X}, \hat{Y}, \hat{Z}\}$. The homogeneous coordinates of the point may be expressed [54]

$$
\mathscr{F}=[X, Y, Z, 1]^{T}
$$

Similarly, a second frame $\mathscr{F}^{\prime}$ with origin $\mathscr{O}^{\prime}$ and right-handed basis $\left\{\hat{x}^{\prime}, \hat{y}^{\prime}, \hat{z}^{\prime}\right\}$ is expressed

$$
\mathscr{F}^{\prime}=\left[x^{\prime}, y^{\prime}, z^{\prime}, 1\right]^{T}
$$

A displacement $\delta=\left[\delta_{X}, \delta_{Y}, \delta_{Z}\right]^{T}$ and rotation $\boldsymbol{\xi}$ of $\mathscr{F}^{\prime}$ with respect to $\mathscr{F}$ may be written as a matrix operation:

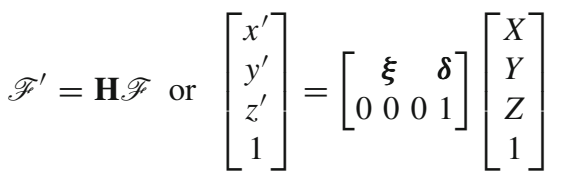

$$
\begin{aligned}
& =\left[\begin{array}{cccc}
c_{Y} c_{Z} & c_{Y} s_{Z} & -s_{Y} & \delta_{X} \\
s_{X} s_{Y} c_{Z}-c_{X} s_{Z} & s_{X} s_{Y} s_{Z}+c_{X} c_{Z} & s_{X} c_{Y} & \delta_{Y} \\
c_{X} s_{Y} c_{Z}+s_{X} s_{Z} & c_{X} s_{Y} s_{Z}-s_{X} c_{Z} & c_{X} c_{Y} & \delta_{Z} \\
0 & 0 & 0 & 1
\end{array}\right]\left[\begin{array}{c}
X \\
Y \\
Z \\
1
\end{array}\right]
\end{aligned}
$$

where $\mathbf{H}$ is the homogeneous transformation matrix [55] and ZYX sequence of Euler angles is assumed to calculate the rotation matrix $\boldsymbol{\xi}$ (see Eq. (23)). The inverse mapping from $\mathscr{F}^{\prime}$ to $\mathscr{F}$ may be expressed:

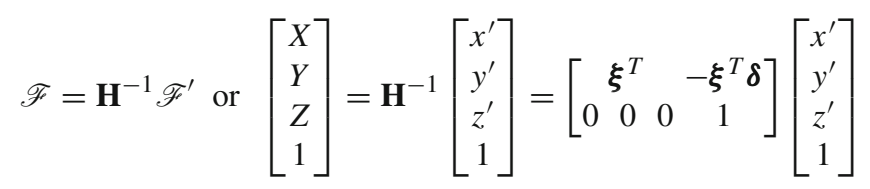


where $\mathbf{H}^{-1}$ is expressed

$$
\mathbf{H}^{-1}=\left[\begin{array}{cccc}
c_{Y} c_{Z} & s_{X} s_{Y} c_{Z}-c_{X} s_{Z} & c_{X} s_{Y} c_{Z}+s_{X} s_{Z} & -\left(c_{Y} c_{Z}\right) \delta_{X}-\left(s_{X} s_{Y} c_{Z}-c_{X} s_{Z}\right) \delta_{Y}-\left(c_{X} s_{Y} c_{Z}+s_{X} s_{Z}\right) \delta_{Z} \\
c_{Y} s_{Z} & s_{X} s_{Y} s_{Z}+c_{X} c_{Z} & c_{X} s_{Y} s_{Z}-s_{X} c_{Z} & -\left(c_{Y} s_{Z}\right) \delta_{X}-\left(s_{X} s_{Y} s_{Z}+c_{X} c_{Z}\right) \delta_{Y}-\left(c_{X} s_{Y} s_{Z}-s_{X} c_{Z}\right) \delta_{Z} \\
-s_{Y} & s_{X} c_{Y} & c_{X} c_{Y} & -\left(-s_{Y}\right) \delta_{X}-\left(s_{X} c_{Y}\right) \delta_{Y}-\left(c_{X} c_{Y}\right) \delta_{Z} \\
0 & 0 & 0 & 1
\end{array}\right]
$$

\section{References}

1. J.C. Maxwell, A Treatise on Electricity and Magnetism (Clarendon Press, Oxford, 1881)

2. K. Tsiolkovsky, Extension of man into outer space, in Proceedings of the Symposium on Jet Propulsion (1921)

3. R.L. Forward, Roundtrip interstellar travel using laser-pushed lightsails. J. Spacecr. Rockets 21, 2 (1984)

4. G. Marx, Interstellar vehicle propelled by terrestrial laser beam. Nature 211, 5044 (1966)

5. J.L. Redding, Interstellar vehicle propelled by terrestrial laser beam. Nature 213, 5076 (1967)

6. W.E. Moeckel, Propulsion by impinging laser beams. J. Spacecr. Rockets 9, 12 (1972)

7. G.A. Landis, Optics and materials considerations for a laser-propelled lightsail, in IAF, 40th International Astronautical Congress, Spain (1989)

8. G.A. Landis, Small laser-propelled interstellar probe, in IAF, 46th International Astronautical Congress, Norway (1995)

9. G.A. Landis, Advanced solar-and laser-pushed lightsail concepts, in NIAC (1999)

10. G.A. Landis, Dielectric films for solar and laser-pushed lightsails, in AIP Conference Proceedings Vol. 504. 1. AIP (2000)

11. E.E. Montgomery IV, Power beamed photon sails: new capabilities resulting from recent maturation of key solar sail and high power laser technologies, in AIP Conference Proceedings, Vol. 1230. 1. AIP (2010)

12. P. Lubin, A roadmap to interstellar flight. J. Br. Interplanet. Soc. (2016)

13. U.S. lawmaker orders NASA to plan for trip to Alpha Centauri by 100th anniversary of moon landing. https://www.sciencemag.org/news/2016/05/us-lawmaker-orders-nasa-plan-trip-alphacentauri-100th-anniversary-moon-landing. Accessed 6 Jun (2020)

14. R.F. Weiss, A.N. Pirri, N.H. Kemp, Laser propulsion. Astronaut. Aeronaut. 17 (1979)

15. D.R. Smith et al., Composite medium with simultaneously negative permeability and permittivity. Phys. Rev. Lett. 84, 18 (2000)

16. E. Schamiloglu et al., 3-D simulations of rigid microwave-propelled sails including spin, in AIP Conference Proceedings. AIP (2001)

17. J. Benford et al., Experimental tests of beam-riding sail dynamics. in AIP Conference Proceedings. AIP (2002)

18. G. Benford, Experimental tests of beam-riding sail dynamics. in AIP Conference Proceedings. AIP (2003)

19. E. Chahine et al., Dynamics and control of microwave-propelled sails, in AIAA Space Conference \& Exposition (2003)

20. M. Polites, J. Kalmanson, D. Mangus, Solar sail attitude control using small reaction wheels and magnetic torquers. Proc. Inst. Mech. Eng. Part G J. Aerosp. Eng. 222, 1 (2008)

21. A. Bedford, W. Fowler, Engineering Mechanics: Statics and Dynamics (Prentice-Hall, Upper Saddle River, 2008)

22. E. Popova, M. Efendiev, I. Gabitov, On the stability of a space vehicle riding on an intense laser beam. Math. Methods Appl. Sci. 40, 4 (2017)

23. Z. Manchester, A. Loeb, Stability of a light sail riding on a laser beam. Astrophys. J. Lett. 837, 2 (2017)

24. R. Gaudenzi, D. Stefani, S.J. Cartamil-Bueno, Light-induced propulsion of graphene-on-grid sails in microgravity. Acta Astronaut. 174, 2020 (2020)

25. A. Ashkin et al., Observation of a single-beam gradient force optical trap for dielectric particles. Opt. Lett. 11, 5 (1986)

26. G.A. Swartzlander, Radiation pressure on a diffractive sailcraft. J. Opt. Soc. Am. B 34, 6 (2017)

27. O. Ilic, H.A. Atwater, Self-stabilizing photonic levitation and propulsion of nanostructured macroscopic objects. Nat. Photonics 13, 4 (2019)

28. P.R. Srivastava, Y.-J. Lucy Chu, G.A. Swartzlander, Stable diffractive beam rider. Opt. Lett. 44, 12 (2019)

29. Y.-J. Lucy Chu, E.M. Jansson, G.A. Swartzlander, Measurements of radiation pressure owing to the grating momentum. Phys. Rev. Lett. 121, 2018 (2018) 
30. Y.-J. LucyChu, N.V. Tabiryan, G.A. Swartzlander, Experimental Verification of a Bigrating Beam Rider. Phys. Rev. Lett. 123, 2019 (2019)

31. H. Sarkissian et al., Polarization-controlled switching between diffraction orders in transverse-periodically aligned nematic liquid crystals. Opt. Lett. 31, 15 (2006)

32. S.R. Nersisyan et al., The promise of diffractive waveplates. Opt. Photonics News 21, 3 (2010)

33. B. Shen et al., Ultra-high-efficiency metamaterial polarizer. Optica 1, 5 (2014)

34. S. Gupta, Single-order transmission diffraction gratings based on dispersion engineered all-dielectric metasurfaces. J. Opt. Soc. Am. A 33, 8 (2016)

35. S.V. Serak et al., Diffractive waveplate arrays [Invited]. J. Opt. Soc. Am. B 34, 5 (2017)

36. Z. Fan et al., Perfect diffraction with multiresonant bianisotropic metagratings. ACS Photonics 5, 11 (2018)

37. O. Ilic, C.M. Went, H.A. Atwater, Nanophotonic heterostructures for efficient propulsion and radiative cooling of relativistic light sails. Nano Lett. 18, 9 (2018)

38. P. Lalanne, J.-P. Hugonin, High-order effective-medium theory of subwavelength gratings in classical mounting: application to volume holograms. J. Opt. Soc. Am. A 15, 7 (1998)

39. C.M. Greiner, D. Iazikov, T.W. Mossberg, Diffraction-limited performance of flat-substrate reflective imaging gratings patterned by DUV photolithography. Opt. Express 14, 25 (2006)

40. T. Clausnitzer et al., Highly-dispersive dielectric transmission gratings with $100 \%$ diffraction efficiency. Opt. Express 16, 8 (2008)

41. W. Sun et al., Multireflection modal method for wideband fused-silica transmission gratings. Appl. Opt. 52, 12 (2013)

42. M. Khorasaninejad, F. Capasso, Broadband multifunctional efficient meta-gratings based on dielectric waveguide phase shifters. Nano Lett. 15, 10 (2015)

43. A. Arbabi et al., Dielectric metasurfaces for complete control of phase and polarization with subwavelength spatial resolution and high transmission. Nat. Nanotechnol. 10, 11 (2015)

44. S. Slussarenko et al., Guiding light via geometric phases. Nat. Photonics 10, 9 (2016)

45. A. Dubill, Attitude Control for Circumnavigating the Sun with Diffractive Solar Sails (2020). MS Thesis (2020). https://scholarworks.rit.edu/theses/10388/

46. L. McNutt et al., Near-Earth Asteroid (NEA) Scout, in AIAA Space Conference and Exposition (2014)

47. B. Dachwald, A. Ohndorf, B. Wie, Solar sail trajectory optimization for the solar polar imager (SPI) mission, in AIAA Astrodynamics Specialist Conference and Exhibit (2006)

48. C.L. Johnson, A.F. Heaton, F.M. Curran, D. Rich, The solar cruiser mission: demonstrating large solar sails for deep space missions. https://ntrs.nasa.gov/search.jsp?R=20190032304. Accessed 4 Jun 2020

49. A.E. Siegman, A.E. Siegman, An Introduction to Lasers and Masers (McGraw-Hill, Cambridge, 1971)

50. T. Kailath, Linear Systems (Prentice-Hall, Upper Saddle River, 1980)

51. A. Bahabad, Diffraction from a moving grating. Opt. Quantum Electron. 46, 8 (2014)

52. D.C. Ullery et al., Strong solar radiation forces from anomalously reflecting metasurfaces for solar sail attitude control. Sci. Rep. 8, 1 (2018)

53. O.M. O'Reilly, Intermediate Dynamics for Engineers: A Unified Treatment of Newton-Euler and Lagrangian Mechanics (Cambridge University Press, Cambridge, 2008)

54. W.M. Newman, R.F. Sproull, Principles of Interactive Computer Graphics (McGraw-Hill, New York, 1979)

55. S. Briot, W. Khalil, Dynamics of Parallel Robots (Springer, Berlin, 2015) 